\title{
Erratum to: Fully automatic segmentation of the Femur from 3D-CT images using primitive shape recognition and statistical shape models
}

\author{
Lassad Ben Younes · Yoshikazu Nakajima · Toki Saito
}

Published online: 29 October 2013

(c) CARS 2013

\section{Erratum to: Int J CARS}

DOI 10.1007/s11548-013-0950-3

The original version of this article unfortunately contained a mistake. The affiliation of the first and second author is incorrect. The correct affiliation for first and second author is mentioned below.

The online version of the original article can be found under doi:10.1007/s11548-013-0950-3.

L. Ben Younes $(\bowtie) \cdot$ Y. Nakajima

Graduate School of Engineering, The University of Tokyo,

Room 307, Takeda Building, Yayoi 2-11-16, Bunkyo,

Tokyo 113-8656, Japan

e-mail: benyounes@image.t.u-tokyo.ac.jp

T. Saito

Graduate School of Medicine, The University of Tokyo, 7-3-1, Hongo,

Bunkyo-ku, Tokyo 113-0033, Japan 